\title{
La universidad recorre la periferia
}

Borello, José Antonio (2004), Argonautas del ConURbano:

DOCENCIA, INVESTIGACIÓN Y EXTENSIÓN EN EL MARCO DE DIAGNÓSTICOS AMBIENTALES MUNICIPALES, INSTITUTO DEL CONURBANO, COLECCIÓN Universidad y EDUCACIÓN, SERIE EXPERIENCIAS EdUCATIVAS NÚM. 3, Universidad Nacional de General Sarmiento, Los Polvorines, Buenos Aires, 114 PP., ISBN: 987-9300-54-8. Información: http://www.ungs.edu.ar/publicaciones/

Con el sugestivo título de Argonautas del Conurbano, el geógrafo José Borello -un reconocido especialista en temas urbanos e industriales de la Argentina- relata en este libro sus experiencias relacionadas con lo que significó para él un verdadero redescubrimiento del paisaje social, ambiental y productivo en los suburbios del noroeste del área metropolitana de Buenos Aires. Desde 1997, el autor se encuentra al frente de una asignatura interdisciplinaria llamada Laboratorio Intermenciones, que se dicta en una universidad estatal de reciente instalación: la Universidad Nacional de General Sarmiento. Borello describe el origen, la 'cocina' y el desarrollo del laboratorio, cuyos integrantes (profesores y estudiantes) deben recorrer cada año un arduo camino de enseñanza-aprendizaje para arribar al fin de la materia: elaborar un diagnóstico urbano-ambiental de un municipio seleccionado y transferir el conocimiento científico generado al medio local.

Este estado de fascinación del autor por el descubrimiento de un mundo nuevo, el de los suburbios, forma parte de un fenómeno mayor. Nos referimos al saludable proceso de descentralización del sistema educativo registrado en la última década en Buenos Aires, como consecuencia de la instauración de nuevas universidades localizadas en la periferia de la aglomeración. Él mismo permitió romper con la impronta centralista que históricamente había tenido para el mundo académico la omnipresencia de la principal casa de estudios de la Argentina, la Universidad de Buenos Aires, localizada justamente en el área central: la Capital Federal (hoy llamada Ciudad Autónoma). Más allá de determinadas circunstancias y objetivos políticos que estuvieron detrás de la formulación de estas instituciones (lo cual merecería una discusión aparte), es indudable que el surgimiento de estas nuevas universidades ha permitido, para diversos investigadores 
y equipos, una significativa ruptura de barreras mentales y geográficas para ponerse en contacto con otras realidades y dedicarse al estudio de los partidos (municipios) de la Gran Buenos Aires.

Es en el marco de este desembarco académico en 'territorio virgen' bonaerense donde el autor comenta cómo el Laboratorio Intermenciones se propuso realizar un diagnóstico de los principales problemas socioambientales de diferentes distritos, y en un contexto donde muchos de los municipios suburbanos prácticamente no cuentan con estudios propios de los territorios sobre los cuales tienen competencia o sus sistemas de información están fuertemente atomizados. Por eso, para producir cada diagnóstico ambiental en el marco de la asignatura, se celebra previamente un convenio entre el municipio de estudio y la universidad. Esto compromete al municipio a brindar la información de la que dispone, y a la universidad, a generar, como devolución, un informe que sistematice los resultados producidos por el laboratorio. Ambas situaciones no están exentas de dificultades. Por el lado de los municipios, las rigideces burocráticas, determinadas prácticas institucionales establecidas y la eterna desconfianza que existe en los organismos municipales hacia 'los de afuera' suelen manifestarse con frecuencia. Y más, si desde el sector político no hay un real convencimiento de la utilidad del trabajo que va a realizarse y simplemente se cumple con un formalismo firmando el convenio. Por el lado de la universidad, y más allá de la saludable declamación de interdisciplina, no siempre resulta sencillo manejar las situaciones interpersonales que se registran entre especialistas provenientes de distintas formaciones. Asimismo, el tiempo con el que cuentan los profesores para preparar a los grupos de estudiantes con miras a realizar las salidas de campo o el tiempo con el que cuentan éstos para investigar y generar los informes finales puede transformarse en un insumo escaso y rápidamente agotable.

Sin embargo, Borello se muestra optimista: una vez establecidas las condiciones de partida, los argonautas del conurbano se lanzan activamente a la concreción de sus objetivos. A través del laboratorio, sostiene, la universidad cumple con sus principios básicos fundamentales: hacer docencia, investigación y brindar servicios penetrando en el medio social circundante. Las encuestas hechas a los alumnos demuestran que la mayoría se siente satisfecha luego de la experiencia que les toca vivir, y que obtienen una mirada mucho más elaborada del territorio que les tocó estudiar. Los estudiantes incorporan la dimensión de que los suburbios que habitan forman parte de un complejo mayor, una 
región metropolitana, lo cual revalora su condición de ciudadanía. A su vez, los docentes, que generalmente provienen de la ciudad de Buenos Aires, se empapan con otras realidades y enriquecen su mirada al estudiar nuevos problemas sociourbanos. Ello colabora significativamente en sus investigaciones, su docencia en otras asignaturas, etc. Finalmente, las barreras intra e interinstitucionales van superándose. El último día de laboratorio, los productos generados por los equipos de docentes y alumnos se exponen y se debaten ante representantes de diversas instituciones locales. Es el momento en el que los argonautas hacen su mise en escene.

Si nos abocamos a un análisis más específico de la estructura del trabajo, podría decirse que tanto las partes más interesantes como las menos se derivan del involucramiento 'desde adentro' que han caracterizado las vivencias del autor. Comenzaremos inicialmente por referirnos a ciertas falencias que, a nuestro entender, presenta el libro.

La introducción a los planes de estudio de la Universidad Nacional de General Sarmiento es sucinta y, para quien no pertenece a la institución, hubiera sido útil la inclusión de un esquema que muestre la centralidad que tiene el Laboratorio Intermenciones como momento de integración final del primer ciclo universitario de todas las carreras. Otra observación está relacionada con la caracterización geográfica de los territorios donde se llevan a cabo las experiencias del laboratorio. Es reducida en extensión. Y la cartografía que se acompaña para mostrar las zonas suburbanas donde se han definido situaciones de distinta criticidad ambiental no resulta del todo clara para el lector, máxime teniendo en cuenta que a partir de allí se realizan varias afirmaciones que describen diferentes situaciones registradas en cada lugar de acuerdo con la dotación de recursos, infraestructuras, usos del suelo permitidos, etc. La cuestión metodológica referida a cómo se establecen zonas ambientalmente homogéneas no está desarrollada. Para quien no conoce Buenos Aires, la comprensión de este punto puede resultar dificultosa. Por otra parte, y yendo más directamente a la experiencia misma, un tema que no está mencionado en el libro es cómo se trabaja el concepto de ambiente en la asignatura y si es que se llega a un mínimo consenso sobre él entre los distintos profesores. Borello menciona una fuerte pugna por la "verdad científica" entre ellos, pero no explica cómo se resuelve el conflicto, ni cómo el término se trata finalmente en los cursos. Asimismo, parecería que el árbol de causas y efectos -un recurso de taller que se utiliza con los gru- 
pos para construir y pactar dentro de los grupos esquemas integradores de los problemas ambientales presentes en cada municipio- constituye una instancia definitiva a la que llegan los alumnos a la hora de realizar sus exposiciones finales. No queda claro si hay un momento de integración posterior; se menciona únicamente que la redacción de los documentos definitivos queda a cargo de los docentes. De todas formas -nobleza obliga-, debe señalarse que muchos de los problemas aquí mencionados son bastante esperables en el marco de una experiencia educativa como la relatada, especialmente porque se trabaja con grupos grandes de profesores y estudiantes de procedencia multidisciplinaria.

Pasemos ahora a lo mejor del libro. Como señala el autor, el estudio consiste en el relato de su experiencia concreta de siete años. Está desprovisto de modismos académicos áridos, típicos de muchas publicaciones de las ciencias sociales. Por lo tanto, presenta un formato liviano, está escrito en un lenguaje accesible y hasta cuenta con una alta dosis de humor ("el Laboratorio es un bicho difícil”; “¿cómo olvidar -recorriendo una zona hostil- lo que se siente ir en el auto y acelerar a fondo mientras llueven los ladrillazos?"; "todos celebran el fin de la cursada... ies la hora del tinto bien ganado!"). Esto permite que cualquier lector o interesado se ponga rápidamente en contacto con las aventuras de Borello y sus argonautas en sus derroteros por José C. Paz, Moreno, Hurlingham y otros suburbios de la Gran Buenos Aires. El libro describe con minuciosidad las fases del armado y desarrollo del laboratorio (discusión y documentos de base, estrategias pedagógicas, objetivos, armado de un plantel docente multidisciplinario, momento de interacción con el municipio, conocimiento previo del terreno, puesta en marcha del dictado de la materia, asignación de tareas, salidas de campo, redacción de informes, etc.). Indudablemente será de gran utilidad para todas aquellas personas e instituciones que, interesadas por fomentar el desarrollo local en sus regiones, estén pensando en replicar experiencias semejantes. Por cómo ha sido pensado, el Laboratorio Intermenciones obliga a la institución universitaria a romper con su endogamia característica y en ese salir al exterior influye sobre el medio social y a su vez es enriquecida acumulativamente, año tras año- por la experiencia. El libro da la pauta de que los objetivos de máxima van cumpliéndose (docencia, investigación, servicios). Y también trasmite las sensaciones del autor, quien muestra permanentemente su fascinación por estar trabajando en las periferias suburbanas en términos de -como diría Bertha Becker- una "frontera experimental”. Más 
allá del hecho de salir al campo y realizar el relevo de la geografía del ambiente en esos territorios, se trata evidentemente de un itinerario con derivaciones hacia la geografía política. En definitiva, "los tripulantes de la nave mítica que recorre una tierra sin mar”, tal como los presenta José Borello, arriban a buen puerto.

ANDRÉS BARSKY Instituto del Conurbano, Universidad Nacional de General Sarmiento (UNGS) correo-e: abarsky@ungs.edu.ar

Andrés Barsky es profesor y licenciado en geografía por la Universidad de Buenos Aires, master en ciencias sociales, mención agrarias por la Facultad Latinoamericana de Ciencias Sociales, sede Argentina. Actualmente es investigador del Área de Ecología Urbana del Instituto del Conurbano en la Universidad Nacional de General Sarmiento (UNGS). Sus líneas actuales de investigación versan en la ecología urbana y agricultura periurbana. También es editor responsable del portal geográfico GeoRed, sitio de información científica y educativa en geografía (Argentina): http://www.georedweb.com.ar/. Entre sus publicaciones se cuentan: "Agricultura periurbana: Diagnóstico socio-ambiental del impacto de las actividades del sector primario del partido de Moreno", trabajo conjunto del Área de Ecología Urbana (UNGS) y el Instituto de Desarrollo Municipal (Municipalidad de Moreno), con Elena Craig y Liliana Falco, conferencia electrónica RUAF CIP SIUPA on Urban Agriculture Methodologies, Resource Centre on Urban Agriculture and Forestry (RUAF), Holanda, 4 al 6 de febrero de 2002. http://www.ruaf.org/conference/methods/papers/ background/barsky.doc; "Sistemas de soporte urbano" (capítulo 5), en M. Di Pace (coordinadora) y H. Caride (editor) Ecología de la ciudad, edición conjunta entre la Universidad Nacional de General Sarmiento y editorial en trámite, Buenos Aires, 2003, con María Di Pace y Alejandro Crojethovich (en prensa); "The Problem of Access to Land of Bolivian Horticultural Producers in the Transition Urban/Rural Zone in the West of Greater Buenos Aires, Focusing Especially on the Partido (Administrative Region) of Moreno. A Geographical Perspective”, enviado para su publicación a The Urban Agriculture Magazine, Resource Centre on Urban Agriculture \& Forestry, AB Leusden, Países Bajos. 



\title{
Volviendo al tema de la democracia
}

\author{
Programa de las Naciones Unidas para el Desarrollo (pnud),
} DaNTE CAPUTO (DIRECTOR DEL PROYECTO) (2004), LA DEMOCRACIA EN AmÉRICA LATINA. HACIA UNA DEMOCRACIA DE CIUDADANAS Y CIUDADANOS, pnud, Aguilar, Altea, Taurus, Alfaguara, Buenos Aires, 255 pp., ISBN: 987-04-0032-9.

En poco más de dos décadas, los países de América Latina fueron adaptando sus regímenes políticos a los preceptos democráticos. No fue este, sin duda, un hecho menor. En la segunda mitad del siglo xx habían arreciado sobre la región cruentas dictaduras que, además de haber contado muertos de a miles, dejaron a la mayoría de los países sumidos en una profunda crisis económica, con una deuda externa impagable, y social. La recuperación, en unos casos, y la instauración de la democracia, en otros, resultó imprescindible para la viabilidad de estas sociedades.

El recibimiento que los latinoamericanos le fueron dando a sus respectivas transiciones no pudo ser más venturoso. El oprobio vivido en las décadas previas había alcanzado su propia cotidianidad, por lo que no se remitía exclusivamente al sistema político. Esto le dio al régimen un bono de legitimidad bastante amplio. No obstante ello, la tarea no era sencilla. Las nuevas democracias debieron enfrentar una doble tensión. Por un lado, las democracias recién instaladas tuvieron que afrontar la tarea de la democratización. Establecer nuevas reglas del juego, modernizar instituciones y procedimientos, dinamizar la vida política, difundir valores democráticos, reinstitucionalizar a los partidos políticos, etc. Por el otro, estaban obligadas a encarar el inmediato problema económico y social. A pesar del bono de legitimidad, las nuevas democracias no podían descansar sólo en ello; requerían, y pronto se notó, eficiencia en la gestión.

La tensión a la que fue sometida la democracia en América Latina, además de las visiones reduccionistas dominantes, hicieron de ella un régimen recluido en lo electoral. El retorno de las democracias a la región se produjo poco después de la crisis de los Estados de bienestar en los países del Primer Mundo. Para entonces el diagnóstico dominante había sido formulado mediante el Informe de la Comisión Trilateral de 1975 denominado La gobernabilidad de la democracia. 
El informe, encargado por dicha comisión a tres destacados politólogos, Samuel Huntington, Michel Crozier y Joji Watanuki, establecía que la crisis que azotaba a los países del Primer Mundo se debía a la sobrecarga de demandas por parte de los ciudadanos hacia el Estado. La concentración en la agenda de actividades que 'debía' desarrollar la sociedad hizo que todas las demandas ciudadanas se dirigieran al Estado, lo que lo orilló a una situación de ingobernabilidad política y anomia social. La solución recomendada se orientaba a la disminución de las expectativas ciudadanas y a la reducción de los servicios sociales del gobierno.

Semejante diagnóstico para las consolidadas democracias centrales no podía más que aterrorizar a las débiles e incipientes democracias latinoamericanas que debían afrontar rezagos sociales mucho más apremiantes. Las recomendaciones de menos democracia, menos política y menos Estado fueron difundidas por los políticos e intelectuales locales de manera inmediata con el objeto de garantizar la gobernabilidad. Los líderes locales se horrorizaron por el peligro de un desbordamiento social que condujera a un retorno del autoritarismo. Así, sociedades acorraladas por sus propias condiciones de subsistencia, que habían luchado por la democracia como una forma de recuperar su propia dignidad, pronto se vieron acorraladas por argumentos que, aunque renovados, sabían a poco democráticos.

El discurso de la gobernabilidad se apoderó también de los organismos multilaterales de crédito, lo cual impactó aún de manera más directa sobre nuestras noveles democracias. El Informe sobre el Desarrollo Humano que el Banco Mundial dio a conocer en 1989, y su impacto por medio de las recomendaciones para la renegociación de la deuda externa y los proyectos de asistencia técnica a los gobiernos de la región, ubicaron el problema del desarrollo en el excesivo tamaño del Estado y en el abultado déficit fiscal que hacían imposible asumir el pago de los compromisos de la deuda y, con ello, el acceso a las indispensables fuentes de financiamiento.

$\mathrm{Al}$ año siguiente, el foro que derivó en el documento denominado Consenso de Washington profundizó esta visión dominante. El foro, al que acudieron académicos y funcionarios públicos de América Latina y de los organismos internacionales, concluyó con diez recomendaciones para superar la crisis de la región, cinco de ellas destinadas a la estabilización económica a través 
de políticas monetarias ortodoxas, y las otras cinco a la reducción sustancial de la dimensión y el papel del Estado.

Esta visión impulsada por los organismos internacionales eliminó del discurso incluso las definiciones en términos de la gobernabilidad, planteadas por el informe de la Comisión Trilateral, que ponía en el centro de la cuestión la crisis fiscal y financiera. Desde principios de la década de los noventa, los problemas vinculados a la definición del Estado y la relación de la economía con el Estado y la sociedad abandonaron la agenda de la democracia en la región para enfocarse exclusivamente en la cuestión monetaria y fiscal.

De tal forma que si para inicios de la transición el dilema giraba en torno a los peligros derivados de los excesos de democracia, para 1990 se centraba exclusivamente en la eficiencia económica del Estado.

La profundidad de la crisis y la contundencia de las recomendaciones obligaron a enfocar todos los esfuerzos gubernamentales y sociales a la política económica. Los encargados de esas carteras, a diferencia de lo ocurrido en épocas previas, se volvieron los personajes clave de sus respectivos gabinetes.

Si bien posteriormente comenzaron a flexibilizarse las recomendaciones de política económica que permitieron una reinterpretación acerca del papel del Estado, lo cierto es que los debates en relación con el papel de la política y la extensión de la democracia no corrieron la misma suerte. ${ }^{1}$ El Estado ideal dejó de ser el mínimo, pero la reconsideración en torno a su dimensión y sus funciones no se planteó en la arena política y ni en la necesidad de acuerdos.

El informe que reseño en esta ocasión es el primer esfuerzo procedente de un organismo internacional que pretende retomar este debate inacabado. Consiste, fundamentalmente, en una reinterpretación de la crisis latinoamericana, tal vez una década más tarde de lo aconsejado, y una recolocación que lleva a plantear el papel de la política y de la democracia en la crisis (que ya deja de considerarse como exclusivamente económica) y en su superación.

Se trata de un documento de poco más de 200 páginas, dividido en tres secciones, además de prólogos, resúmenes y apéndices, en el que se plantean tres preguntas fundamentales: ¿Cuál

${ }^{1}$ El Informe sobre el Desarrollo Humano que el Banco Mundial presentó en 1993 y el informe denominado El Estado en un mundo en transformación que el mencionado organismo publicó en 1997 son prueba de ello. 
es el estado de la democracia en América Latina? ¿Cuáles son las percepciones y cuán fuerte es el apoyo de líderes y ciudadanos a la democracia? ¿Cuáles serían los principales temas para un debate orientado a lograr un mayor avance en la democracia de ciudadanos?

Para responder a la primera pregunta, el informe parte de una definición de democracia como forma de organización social que tiene su fundamento normativo en la definición del ser humano como sujeto portador de derechos y como ciudadano, fuente de autoridad, sobre quien descansa la soberanía popular que se expresa en elecciones libres e institucionalizadas. Se trata de un sujeto responsable y autónomo, de un individuo capaz de elegir, quien, en tanto ciudadano y fuente de la que emana toda autoridad, no puede ser tratado como súbdito.

Hasta aquí la definición no se aparta de la que postulaba el liberalismo decimonónico, y resulta muy acorde con los planteamientos de los defensores del Estado mínimo, si no fuera que considera al sujeto no sólo como portador de derechos políticos, sino también como portador de derechos que hacen posible tales derechos políticos; y a la democracia como un régimen político que requiere, para ser tal, derechos políticos pero también derechos civiles (fundamentalmente la libertad para elegir y la equidad) sin los cuales la ciudadanía es superficial, y sociales, definidos como la base de condiciones para eliminar las privaciones que impiden el ejercicio de las opciones responsables y las libertades que implican.

Desde esta perspectiva, la democracia no tiene sólo una dimensión institucional. Las reglas que implica el régimen político democrático son sólo condiciones necesarias pero no suficientes para hablar de democracia. La democracia va más allá del régimen político

A partir de su definición, el informe intenta describir la situación actual de la democracia en América Latina mediante un conjunto de indicadores que abarcan cada una de las dimensiones de la ciudadanía integral.

Los indicadores de desarrollo de la ciudadanía política se encuentran descritos en el cuadro 1. El balance que arroja la región en relación con estos indicadores es, de acuerdo con el informe, bastante positivo en la medida que: 1) es ampliamente reconocido el derecho al voto; 2) la práctica de las elecciones limpias constituye un patrón generalizado; 3) la imposición de las elecciones como medio de acceso a cargos públicos registra un nota- 
ble avance; 4) la participación electoral es moderadamente amplia; 5) no existen marcadas barreras de acceso para la competencia política; 6) se percibe una tendencia positiva a la introducción de normas para una mayor inclusión, y 7) existe una considerable proporcionalidad en los sistemas electorales. Los aspectos marcados como pendientes de atención son: a) el corto alcance de la legislación sobre el financiamiento de los partidos; b) la persistente supremacía del Poder Ejecutivo sobre el Legislativo; c) las interferencias sobre el Poder Judicial; $d$ ) el desarrollo desigual de los mecanismos de democracia directa, y e) la poca independencia de las agencias especializadas en el control de la gestión pública.

\section{Cuadro 1}

\begin{tabular}{lll}
\hline Índice de democracia & $\begin{array}{l}\text { Elecciones libres } \\
\text { electoral } \\
\text { Elecciones limpias } \\
\text { Cargos públicos electos }\end{array}$ \\
Ciudadanía política & Control de gestión & $\begin{array}{l}\text { División de poderes } \\
\text { Democracia directa } \\
\text { Agencias especializadas }\end{array}$ \\
& $\begin{array}{l}\text { Clecciones internas en los } \\
\text { partidos }\end{array}$ \\
selección de candidatos & $\begin{array}{l}\text { Barreras de entrada a los } \\
\text { partidos políticos } \\
\text { Monopolio partidista de las } \\
\text { candidaturas }\end{array}$ \\
\hline
\end{tabular}

El cuadro 2 resume los indicadores que utiliza el informe para la evaluación del desarrollo de la ciudadanía civil. Los resultados de la valoración de esta dimensión de la ciudadanía integral son menos alentadores que en los de la dimensión anterior. Los datos aportados por el informe apuntan a: 1) ciertos logros significativos en el reconocimiento legal de los derechos civiles en general, de las mujeres y de los pueblos indígenas; 2) avances en el respeto a los derechos humanos y la libertad de prensa; 3) disparidades en la aplicación de la igualdad legal y la protección contra la discriminación; 4) altos niveles de inseguridad ciudadana que limitan el derecho a la vida, a la integridad física y a la seguridad, y 5) violaciones de los derechos de los acusados y los presos que no evita el funcionamiento de la administración de justicia. 


\section{Cuadro 2}

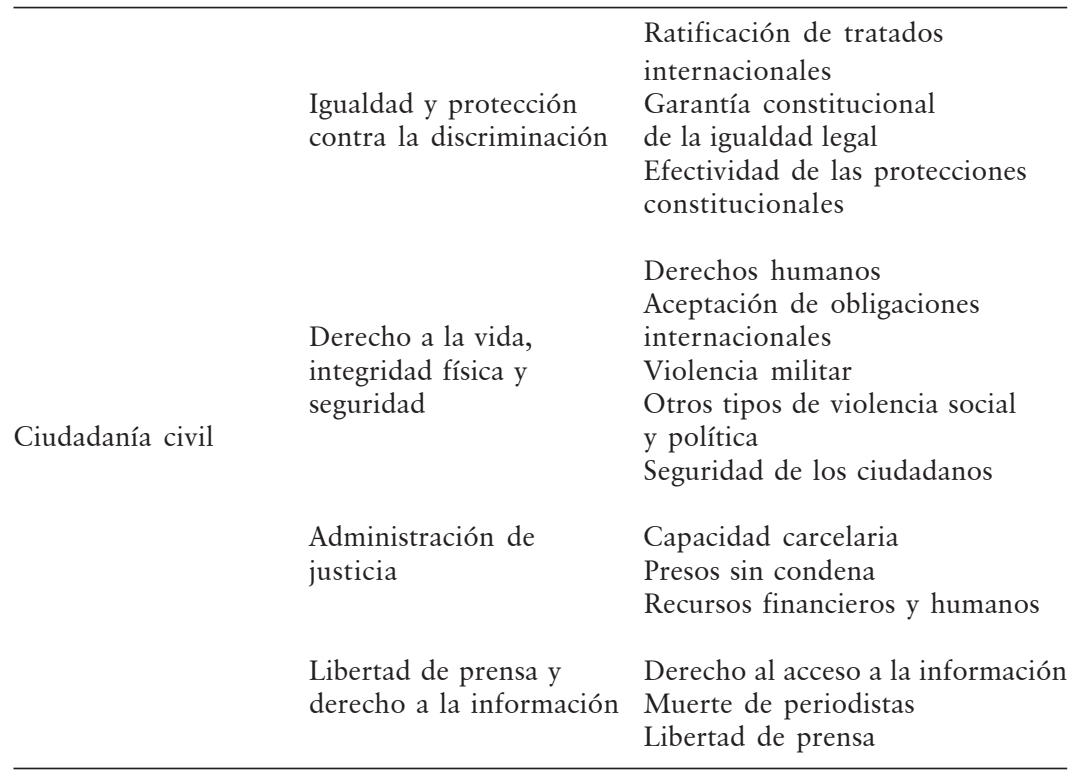

Por lo que se refiere a los derechos sociales, los datos aportados para los indicadores utilizados por el informe, resumidos en el cuadro 3, muestran que esta es la dimensión de la ciudadanía integral que peores condiciones registra, por lo que se considera en el documento que es uno de los desafíos más urgentes que enfrenta la región.

\section{Cuadro 3}

\begin{tabular}{lll}
\hline \multirow{2}{*}{ Ciudadanía social } & Integración social & $\begin{array}{l}\text { Pobreza } \\
\text { Desigualdad } \\
\text { Empleo }\end{array}$ \\
& Necesidades básicas & $\begin{array}{l}\text { Educación } \\
\text { Salud }\end{array}$ \\
\hline
\end{tabular}

Para responder la segunda pregunta que se plantea el informe, se realizó una encuesta durante el mes de mayo de 2002 sobre las percepciones ciudadanas acerca de la democracia, así como una ronda de consultas a 213 líderes latinoamericanos, incluidos 41 presidentes y vicepresidentes.

Los resultados obtenidos de la aplicación del primer instrumento son los siguientes: 1) 43\% de los encuestados tenían una 
orientación prodemocrática, es así la orientación más extendida; 2) la alternativa entre desarrollo económico y democracia evidencia una tensión, y parecería que muchos prefieren la primera; 3) los entrevistados pertenecientes a países donde hay menores niveles de desigualdad social tienden a ser menos favorables a la democracia; 4) los ciudadanos con orientaciones no democráticas se encuentran mayoritariamente entre los sectores que tienen menor educación, una socialización proveniente de periodos autoritarios, una percepción de baja movilidad social respecto de sus padres y bajas expectativas en cuanto a una futura mejoría para sus hijos, y aquellos que tienen mayor desconfianza en las instituciones; 5) la mayoría de los ciudadanos no está desconectada de la vida política y social de sus países, y 6) en promedio, los demócratas tienden levemente a participar más activamente en la vida política de sus países.

Por su parte, la ronda de entrevistas a los líderes muestra las siguientes opiniones preponderantes: 1) América Latina ha dado pasos muy importantes en el camino hacia la democratización, el aumento de la participación y de los controles institucionales es reconocido como un paso decisivo; 2) toda la región es, al menos formalmente, democrática, la dimensión institucional no se ve como un epifenómeno de lo que realmente importa, sino como parte constitutiva de la democracia; 3) algunas amenazas tradicionales a las democracias latinoamericanas han desaparecido o se han debilitado significativamente; 4) han aparecido otras amenazas que siguen poniendo en cuestión la continuidad y expansión de la democracia, la más ostensible es el narcotráfico; 5) otras amenazas sobre la democracia latinoamericana son políticas, las más importantes son: la reducida autonomía de decisión de los poderes institucionales y el debilitamiento de los partidos políticos; 6) la crisis de los partidos no ocurre debido a una pérdida de la voluntad ciudadana de participación, antes bien, enfrentan un problema nuevo que combina tres elementos: una voluntad de mayor participación y control de poder político, un rechazo generalizado de los partidos como canales de participación y un traslado de la participación y el ejercicio de controles hacia otros tipos de organizaciones en general pertenecientes a la sociedad civil; 7) es importante tener partidos fuertes y gobiernos con capacidad de decisión; 8) un primer desafío de la democracia latinoamericana es encontrar soluciones políticas a sus problemas políticos; 9) un segundo desafío es encontrar soluciones a la desigualdad, la pobreza y la actual imposibilidad de 
acceso de gran parte de la población a los niveles de bienestar necesarios para el pleno ejercicio de los derechos.

Aportados los datos que arroja el estudio empírico, el informe se aboca a la definición de cuatro esferas que, en su evaluación, resultan indispensables para el desarrollo de la democracia en América Latina: política, Estado, economía y globalización.

El informe establece que existe un déficit de poder democrático en las democracias de la región. Define este poder como la capacidad de actuar de modo efectivo frente a los problemas para expandir la ciudadanía. Sostiene, asimismo, que para construirlo es indispensable la política: "que la política sea relevante, que proponga caminos para abordar los temas claves de la sociedad, que los emprenda con la firmeza de la voluntad de los líderes y ciudadanos y la sostenga con la idoneidad de los instrumentos para la acción colectiva, entre los cuales los partidos políticos son actores centrales pero no los únicos” (p. 181).

El informe propone que las democracias latinoamericanas tienen también un déficit de estatalidad. Las propuestas de los partidos y de los actores de la sociedad civil requieren la eficacia ejecutoria de las instituciones representativas y del gobierno del Estado. Pues "el poder democrático se construyen también desde la estatalidad. Detrás de todo derecho hay un Estado que lo garantiza. Y detrás de todo derecho trunco hay un Estado que no llega a tornarlo efectivo. Esta defección del Estado tiene que ver con la calidad de sus instituciones y, fundamentalmente, con el poder que fluye a través de ellas y la consecuente capacidad -O incapacidad- del Estado para llevar a cabo sus fines” (p. 181).

Los déficit de política y de estatalidad que postula el informe se combinan con los requerimientos de crecimiento económico para superar los rezagos documentados por el informe en materia de ciudadanía social y con otros desajustes igualmente analizados. El crecimiento económico no es un dato sin más, sus resultados, frecuentemente generadores de desigualdades, conjugado con los límites del Estado, "con la impotencia de la política para encarar las aspiraciones de la ciudadanía en poder democrático, con las tensiones de sociedades fracturadas, con la existencia de poderes fácticos que evaden la legalidad, trafican influencias y permean las más altas instancias de decisión, con la evidencia de una globalización que acota el espacio propio de la democracia al escamotear del campo de la voluntad ciudadana los temas centrales que atañen al futuro de la sociedad" (p. 182). 
En América Latina, donde los principales desequilibrios se manifiestan en la dimensión social de la ciudadanía, "la sustentabilidad del sistema y la resolución de la crisis de representación política dependen de nuestra capacidad para incorporar a la economía y sus opciones como un tema de la democracia [...] La economía es una cuestión de la democracia porque de ella depende el desarrollo de la ciudadanía social y porque genera y altera las relaciones de poder” (p. 191). Y viceversa:

existen cinco funciones que las instituciones públicas deberían ofrecer para que los mercados funcionen adecuadamente: la protección de los derechos de propiedad, la regulación del mercado, la estabilización macroeconómica, el seguro social y el manejo de los conflictos de interés [...] Los mercados requieren gobernabilidad y reglas. La buena gobernabilidad sólo se asegura por la vía de la democracia [...] por ello la agenda de la sustentabilidad democrática debe incluir, a riesgo de vaciarse de contenido, estas cuestiones de la economía, sus opciones y su diversidad (p. 192).

El último tema de la agenda planteado por el informe es el de la globalización. Al respecto señala que el debate se ha centrado en los asuntos financieros y comerciales, y margina el hecho de que con la globalización los poderes exteriores han dejado de ser exteriores; que condicionan las decisiones del Estado más allá de los ámbitos financiero y comercial; que abarcan las cuestiones políticas, de seguridad, de seguridad social, de educación y de salud. Por esta razón, prosigue, es necesario observar, por un lado, el impacto real en términos de la soberanía interior de los Estados y, por el otro, concebir las estrategias posibles para aumentar las capacidades nacionales y regionales, para que el poder nacional no se extinga en nombre de un incontrolable poder global.

En suma, el informe llama a fortalecer la democracia mediante: 1) la revaloración de la política; 2) la recuperación del papel constructivo de la política como ordenadora de las decisiones de la sociedad; 3) la expansión de la ciudadanía; 4) el robustecimiento de la participación ciudadana; 5) sistemas de partidos que se fortalezcan a partir de la eficacia, la transparencia y la responsabilidad; 6) el fortalecimiento de la sociedad civil; 7) un Estado capaz de conducir el rumbo general de la sociedad; 8) una economía congruente con la democracia, y 9) la construcción de espacios autónomos. 
Es, sin duda, loable el esfuerzo de recuperar el debate sobre la democracia en la región. Mucho más cuando se hace desde un organismo internacional, lo cual implica una posición como interlocutor mucho más influyente que la que se tiene desde la academia. Independientemente de los responsables de llevar a cabo este informe, lo cierto es que, desde el punto de vista de la institución que lo produce, es un esfuerzo tardío. El daño hecho en la década de los noventa a los países de la región es irreparable. No sólo sus consecuencias más connotadas son de por sí lamentables, su perjuicio fue igualmente grave sobre las mediaciones que hacen posible la vida democrática. Por supuesto que, como dice el refrán, más vale tarde que nunca; pero no basta con cambiar de perspectiva, resulta necesario asumir las responsabilidades de lo realizado anteriormente. No será posible el desarrollo de la ciudadanía integral sin condiciones especiales para su recuperación. Se requiere un esfuerzo y un compromiso de parte de este tipo de instituciones que todavía no se ha percibido con claridad, ni el informe aborda con nitidez y compromiso.

Como trabajo académico, el informe tiene, insisto, la virtud de reubicar el debate. No se trata de un trabajo totalmente original en este esfuerzo. La academia viene advirtiendo sobre los problemas de la consolidación democrática en la región desde hace ya tiempo, aunque, como se dijo, con un impacto muy limitado. Incluso desde el punto de vista del análisis mismo, se requiere documentar más eficientemente muchas de las afirmaciones vertidas. Vale, pues, tanto por lo que dice como por quien lo dice. Y esto no es poco.

Javier Arzuaga Magnoni Facultad de Ciencias Políticas y Administración Pública, UAEMéx

Correo-e: jaam@uaemex.mx

Javier Arzuaga Magnoni es doctor en ciencias sociales por la Universidad Autónoma Metropolitana-Xochimilco, y miembro del Sistema Nacional de Investigadores. Desde 1993 se desempeña como investigador del Centro de Estudios Avanzados de la Facultad de Ciencias Políticas y Administración Pública de la Universidad Autónoma del Estado de México. Sus líneas de investigación son competitividad y geografía electorales así como sistemas de partidos políticos en México. Es autor del libro Racionalidad empresarial. Los megaempresarios mexicanos, coeditado 
por Gernika y la Universidad Autónoma del Estado de México en 2004; en 2002 publicó en coautoría con Carlos Alberto Sara Gutiérrez "La alianza virtual. Razones del cambio en las elecciones presidenciales en el Estado de México", Economía, Sociedad y Territorio, El Colegio Mexiquense, A.C., vol. III, núm. 11, Zinacantepec, pp. 441-470; y en 2000 publicó en coautoría con Cecilia Gayet "El avance de la pluralidad electoral en los sistemas políticos locales del Estado de México", Apuntes Electorales, revista del Instituto Electoral del Estado de México, año 1, núm. 2, Toluca, pp. 191-200; asimismo, "Democracia y consenso: una apuesta al gobierno de lo local”, Espacios Públicos, año 4, núm. 7, febrero de 2001, Toluca, México; "Argumentos a favor de una democracia con adjetivos", Apuntes Electorales, año 1, núm. 3, diciembre de 2000, Toluca; y “1996: Elecciones municipales y legislativas”, en coautoría con Ernesto Emmerich en Ernesto Emmerich (coord.), El voto ciudadano en el Estado de México (1990-1997), Universidad Autónoma del Estado de México, Toluca. 



\title{
Lecturas en contraste. El lector frente a un texto
}

\author{
Castro, Maricruz, Laura Cázares y Gloria Prado (editoras) \\ (2004), ESCRITURAS EN CONTRASTE. FEMENINO/MASCULINO EN LA \\ LITERATURA MEXICANA DEL SIGLO XX, UNIVERSIDAD AUTÓNOMA \\ Metropolitana Unidad IzTapalapa/ aldus, MÉxico, 366 PP., ISBN: \\ 970-714-070-4.
}

Este libro contiene 12 ensayos escritos por mujeres, académicas especializadas en el campo de las letras, unas mexicanas nacidas en la ciudad de México y otras en ciudades como Mérida, Coatzacoalcos y Toluca; las menos nacidas en otros países y ciudades como Córdoba, Argentina. Las autoras son, en orden alfabético: Enid Álvarez, Regina Cardoso Nelky, Maricruz Castro Ricalde, Laura Cázares, Ana Rosa Domenella, Luzelena Gutiérrez de Velasco, Graciela Martínez-Zalce, Nora Pasternac, Gloria Prado, Berenice Romano Hurtado, Ute Seydel y Luz Elena Zamudio Rodríguez.

El libro, como indica la introducción, es producto del Taller de Teoría y Crítica Literaria Diana Morán-Coyoacán que inició sus labores bajo los auspicios del Programa Interdisciplinario de Estudios de la Mujer de El Colegio de México y que en 1993 se constituyó como grupo de trabajo independiente. Su línea principal de estudio es la literatura escrita en México y en América. El grupo reúne a un poco más de 20 académicas, la mayoría con formación en el área de la literatura, procedentes de instituciones diferentes como la Universidad Nacional Autónoma de México, la Universidad Autónoma Metropolitana, Unidad Iztapalapa; el Instituto Tecnológico Autónomo de México, El Colegio de México, la Universidad Iberoamericana, la Universidad Autónoma del Estado de México y el Instituto Tecnológico y de Estudios Superiores de Monterrey, campus Toluca. Académicas que se reúnen mensualmente desde hace 18 años echando por tierra el dicho de 'mujeres juntas, ni difuntas'. Esta publicación, lo mismo que algunas anteriores y seguramente otras que vendrán, demuestra el trabajo creativo de las mujeres cuando trabajan en grupo.

Desde la introducción de este libro, y haciendo referencia a Borges, se advierte que censurar y alabar son operaciones sentimentales que nada tienen que ver con la crítica, entonces desde 
la entrada se avisa que el mayor aporte que puede hacerse a un texto escrito no es la censura ni la alabanza infundadas, aunque después de leer cuidadosamente la obra puedan venir ambas, pero fundamentadas en un trabajo de análisis. Esto es lo que me propuse hacer en este comentario.

Antes de entrar a la obra, me detuve en el título, especialmente en la palabra contraste. Para empezar a elaborar mis juicios, consulté el diccionario de filosofía de Ferrater Mora y encontré: contraste-asociación. Aristóteles fue de los primeros filósofos que definió este concepto lógico al aceptar contraste como comparación-asociación por semejanza o por contigüidad. También encontré contraste como conexión entre diferentes pensamientos e ideas y que mantienen cierto método y regularidad con un interés predominantemente epistemológico. En los ensayos de este libro se aplicaron las dos principales asociaciones: por semejanza que lleva implícita la diferencia, y por contigüidad en tiempo y espacio; además es evidente el fuerte interés epistemológico que posee y que marca la búsqueda del conocimiento y la comprensión. Luego en este libro el término contraste no fue utilizado en el sentido de oposición y hasta de exclusión, sino en el de estudio comparativo buscando conexiones entre ideas y resultados de escritores diferentes por su sexo, pero que compartieron temas y preocupaciones propios de todo ser humano, más allá de consideraciones sexistas.

Los ensayos son diversos en sus contenidos, poco distintos en su extensión. Los autores abordados en cada uno son también diferentes, excepto en el caso de Berenice Romano y Gloria Prado, quienes se ocupan igualmente de Carlos Fuentes en el caso del escritor, pero tomando por pareja de comparación a escritoras distintas. Los autores 'pareados' en los análisis fueron: Elena Garro (n. en 1916 en Puebla) y Juan Rulfo (n. en 1918 en Sayula, Jalisco), Ute Seydel compara de manera especial dos novelas: Los recuerdos del porvenir y Pedro Páramo. Carmen Rosenzweig (n. en 1926 en Toluca, México) y Sergio Galindo (n. en 1926 en Jalapa, Veracruz), Maricruz Castro Ricalde estudia sus obras 1956 y Polvos de arroz. Amparo Dávila (n. en 1928 en Pino, Zacatecas) y Juan José Arreola (n. en 1918 en Zapotlán, Jalisco), Regina Cardoso Nelky analiza los relatos La señorita Julia y La migala. Elena Poniatowska (n. en 1932 en París) y Carlos Fuentes (n. en 1928 en Panamá), Berenice Romano Hurtado compara las novelas autobiográficas Flor de lis y Diana o la cazadora solitaria. Aline Pettersson (n. en México en 1938) y Carlos Fuentes, Glo- 
ria Prado analiza sus novelas Piedra que rueda y Los años con Laura Díaz. Inés Arredondo (n. en 1928 en Culiacán, Sinaloa) y Sergio Pitol (n. en 1933 en Puebla), Laura Cázares aborda sus cuentos Río subterráneo y Victorio Ferri cuenta un cuento. Julieta Campos (n. en 1932 en La Habana) y Salvador Elizondo (n. en 1932 en la ciudad de México), Luzelena Gutiérrez de Velasco analiza algunos de sus ensayos. Angelina Muñiz (n. en 1936) y Federico Patán (n. en 1937) ambos españoles emigrantes, Luz Elena Zamudio Rodríguez compara sus relatos autobiográficos como Seudomemorias y Autorretrato lingüístico. Elsa Cross (n. en 1946 en la ciudad de México) y José Emilio Pacheco (n. en 1939 en la ciudad de México), Nora Pasternac contrasta sus obras de poesía más recientes, Cantáridas y Siglo pasado (Desenlace). María Luisa Puga (n. en 1944 en la ciudad de México) y Luis Arturo Ramos (n. en 1947 en Minatitlán, Veracruz), autores de la novela La viuda y del cuento La señora de la Fuente, son estudiados por Ana Rosa Domenella. Paloma Villegas (n. en 1951) y Héctor Manjarrez (n. en 1945), autores de las novelas La luz oblicua y Pasaban en silencio nuestros dioses, son abordados por Enid Álvarez. Finalmente Susana Pagano (n. en 1968 en la ciudad de México) y Álvaro Enrique (n. en 1969 en la ciudad de México), autores de las novelas Trajinar de un muerto y La muerte de un instalador, son estudiados por Graciela Martínez-Zalce.

Infiero que determinó el orden de los ensayos el año de nacimiento de cada escritor y escritora abordados, autodidactas algunos como literatos, tal es el caso de Amparo Dávila y Juan José Arreola, otros académicos de las letras y del periodismo, pero todos reconocidos como destacados representantes de la literatura mexicana del siglo xx, que es el subtítulo de esta obra. Escritores galardonados casi todos ellos, con premios que incluso llevan sus nombres, como el Premio Xavier Villaurrrutia, Premio Juan Rulfo, Premio Nacional de Literatura, Premio Nacional de Letras, Premio Juan Ruiz de Alarcón, Premio Nacional de Narrativa, Premio Planeta, Premio José Revueltas, Premio Joaquín Mortiz, Premio Nacional de Novela, incluso el Premio Cervantes, otorgado por los monarcas españoles.

Los temas que se abordan en estos trabajos y que sirven como punto de contraste-comparación son diversos. Estos contrastes me permitieron algunas primeras reflexiones de las que parten las autoras de la introducción: Maricruz Castro Ricalde, Laura Cázares Hernández y Gloria Prado Garduño, quienes además 
hacen una muy buena reseña de cada uno de los trabajos que incluye el libro.

Una de las primeras reflexiones a las que invita el libro desde su introducción es la relación entre la historia y la literatura, que a veces se han considerado como antagónicas; recordemos a Aristóteles, quien en La poética defendió la superioridad de la literatura frente a la historia, pues la primera cuenta las cosas como 'ojalá hubieran sucedido', gracias a la imaginación y la ficción creativas del escritor; en cambio la historia, según el estagirita y posteriormente los positivistas franceses del siglo XIX encabezados por Comte, cuenta las cosas 'tal como pasaron', aunque definitivamente hoy este concepto de historia neutral y positiva no existe.

Otro tema de reflexión es el papel de la ficción en la literatura. La ficción puede ser entendida como las cosas arregladas, modeladas, disfrazadas y con ello convertidas en 'apariencia', instrumento necesario para cierta forma del saber: ¿la literatura? No será la apariencia lo que fue el fenómeno para Kant, "la cosa para nosotros", ante la imposibilidad de conocer la "cosa en sí", nóumeno. Luego ¿todo es apariencia?, y si esto es así, ¿serán la lógica kantiana y la literatura las que ordenan el caos que es la realidad aparente que nos ofrecen nuestras percepciones, aunque el saber pleno y absolutamente verdadero no se alcance nunca y sea inaccesible al ser humano? Bergson afirmó que precisamente la literatura presenta la síntesis del conocimiento humano, en la intuición creadora ocurre la inmediatez del instinto y la distancia de la inteligencia. Gracias a la intuición, afirmó Bergson, vivimos dentro y comprendemos fuera. Esto sucede plenamente en la literatura, como lo muestran los 12 ensayos de este libro.

El problema de la hermenéutica también se plantea en la entrada de esta obra. La hermenéutica que desde Platón es aceptada como interpretación que comunicamos; un mismo término implica no sólo significados distintos, sino a veces hasta antagónicos expresados a través de símbolos de una realidad que es necesario interpretar y comunicar. Un trabajo hermenéutico de crítica temática comparada fue lo que hizo cada una de las profesionales que participaron en este libro. Todos estos asuntos planteados en la introducción subyacen y acompañan a los ensayos de este libro, pero ¿̇uáles son algunos temas que de manera explícita aparecen en este contraste de lecturas de escritos por hombres y por mujeres? 
Uno de ellos es la lucha armada de 1917 y la guerra cristera de los años veinte, temas sobre los que escribieron Elena Garro y Juan Rulfo, quienes elaboraron un contradiscurso en la literatura, cuestionaron la historia oficial, la llamada "de bronce" por Luis González entre otros. Luis González, recién fallecido, en su Pueblo en vilo hizo hablar a quienes su historia no importa para la historia oficial, igual que Garro y Rulfo. Ambos mezclan realidad y ficción, muestran unos personajes no nacionales: es manifiesta la ausencia de ideales claros en quienes participaron en los diversos bandos revolucionarios, lo mismo que expuso Azuela en Los de abajo; el cambio de bandos, la invocación de los santos, vírgenes y toda imagen religiosa capaz de hacer milagros, cada quien para su bando y contra el opositor. Garro escribió en Los recuerdos del porvenir la invocación de un cristero: "iVirgen de Guadalupe, ayúdanos a chingar a estos cabrones!”

Junto a estos temas está la ausencia de una nación y un Estado fuertes y consolidados; la brutalidad de los de arriba como Carranza, Villa u Obregón, lo mismo que los de abajo. También está presente la crítica al poder absoluto y corrompido.

El mito del campo y del campesino idílicos, semejantes al paraíso perdido, el campo virgen y noble frente a la feroz y viciosa ciudad son otros asuntos que están presentes en las obras analizadas; la actitud paternalista del mestizo hacia el indio; la aparición de los considerados sin historia, la voz de los personajes marginales, la exclusión de las mujeres, las sin apellido, apenas un nombre si no es que un apodo, mujeres "menos vivas que un cadáver”. Ellos, los hombres, ellos sí con nombre y apellido.

La sexualidad, la rebelión social, el cuerpo, el alcoholismo y otros temas prohibidos entre 'la gente de bien', la estrechez de la provincia frente al mundo multiforme y variado de las ciudades, la hipocresía de los pueblos chicos donde 'todos se conocen', el mercado y la iglesia, la cantina y el burdel, el anonimato de las ciudades, los mundos de los hombres y de las mujeres, la vejez y la muerte, la soledad y la pobreza como elementos existenciales de todos los protagonistas de las obras analizadas.

La neurosis y la angustia como fuentes del arte a la manera de Nietzsche y Freud, la necesidad de escribir para salir de sus propias depresiones, los animales (arañas y rinocerontes) como recipientes de nuestros mundos y locuras y el temor a su dominio y falta de control frente a la luz y la esperanza que significa vivir.

Las aparentes dicotomías y luchas de opuestos, en un permanente tejido de contrarios: caos-orden, noche-día, ciudad-cam- 
po, revolución-evolución, locura-cordura, hombre-mujer, violencia-amor, atracción-repulsión. Yo misma dudé dónde colocar los extremos de estos pares antitéticos en las lecturas analizadas por las autoras de estos libros; y en sus ensayos, no se los ubica, se habla de ellos pero no se los coloca en algún extremo; así aparece la literatura como síntesis enriquecida de un fino tejido de contrarios.

Otro tema es la dualidad en la que se mueve todo ser humano. Freud aparece en el fondo hablándonos de nuestros temores acallados y hundidos en nuestro inconsciente y, como el analista, la literatura colocándolos frente a nosotros para tener conciencia de ellos.

El encuentro de las parejas no sólo en el amor, sino especialmente en la locura y la violencia. La degradación de la mujer casi como la expone Erasmo de Rotterdam (siglo XVI):

\footnotetext{
Cuando alguna mujer quiere pasar por inteligente, solamente pone en evidencia su locura. Es como si se soltara un buey en los serenos dominios de Minerva. Cualquiera que haciendo violencia a su propia naturaleza pretende cubrirse por apariencias de virtud y talento, no hace más que poner en evidencia sus defectos. Dice un proverbio griego: "Aunque la mona se vista de seda mona se queda" La mujer es mujer, es decir loca, sea cual sea el disfraz que adopte.
}

Algunos de los autores abordados en los 12 ensayos de este libro no vivieron la realidad relatada en sus obras, sino que la escucharon desde un plano externo; otros, por el contrario, tienen una obra fundamentalmente autobiográfica como Fuentes, Poniatowska, Rosenzweig, Garro, Rulfo, Galindo y algunos más, ¿̇o son todas obras autobiográficas? Obras donde no fue el yo del autor el que determinó su autobiografía sino al revés, según lo dice Berenice Romano. La realidad y la ficción del escritor construyendo su autobiografía en la que se unen lo público y lo privado, la historia objetiva y las historias personales, el tiempo histórico y el tiempo subjetivo donde, como escribe Gloria Prado, todo quedó unido en la literatura como dos caras de una misma realidad, no como dicotomías sino como una unidad de contrarios. Aparece el ser humano como persona unitaria, suma -no acumulación de partes- de múltiples relaciones no unívocas sino integradas por una compleja red de relaciones e interdependencias donde "yo soy yo y mi circunstancia", como enunció Ortega y Gasset, y si me salvo yo se salva ella. 
La muerte rondando en todas las obras analizadas y el hombre, como refiere Domenella, teniendo conciencia de ella, como lo establecieron Sartre, Kierkergaard, Unamuno y otros existencialistas más; y todos nosotros fingiendo que vivimos por siempre.

Las preocupaciones de todo ser humano: la rutina, el posmodernismo, el nihilismo a la nietzscheano como absoluta aniquilación, la propuesta de androginizar los sentimientos y sus manifestaciones como una alternativa salvadora para hombres y mujeres. En las obras contrastadas se manifiesta el ser humano, hombre y mujer, en toda su fragilidad y en toda su grandeza, como Mefistófeles en el Fausto de Goethe, quien dice:

Soy el espíritu que siempre niega.

Y ello con razón, pues todo lo que nace

No vale más que para perecer.

Por eso sería mejor que nada surgiera.

O a la manera de Calderón en La vida es sueño cuando Segismundo declara:

Pues el delito mayor

Del hombre es haber nacido...

De estos sentimientos de nulidad, como lo hicieron el mismo Goethe y Calderón, se ha logrado la producción literaria, que prueba lo dicho por Bakunin de que sólo la destrucción es creadora.

La palabra escrita como forma de renacimiento y autoconstrucción, eso hicieron las autoras de estos ensayos. Las cartas, los discursos, los cuentos, los poemas, las novelas, los ensayos fueron los materiales de sus trabajos para entregarnos finalmente 12 ensayos; ensayos a la manera de Montaigne, quien utilizó esta forma literaria para expresar sus intuiciones sobre el hombre y la vida, cuestiones que hoy llamamos "existenciales" y que algunos consideraron como 'filosofía subjetiva'; hoy nada más lejos de eso, como lo muestran las 12 escritoras de este libro.

Las autoras dieron a conocer, aunque sea parcialmente, sus preferencias y saberes, además de los mundos de los escritores seleccionados, entonces son varios mundos los que este libro ofrece. Nada de lo que aquí se aborda puede sernos indiferente y, junto con Montaigne, podemos decir: "Todo hombre -yo agrego: y mujer- lleva en sí el peso de la humana condición”, luego nada de lo humano nos puede ser ajeno, y fue precisamente el mundo de lo humano lo que en este libro se aborda. 
Como lo dije en las primeras líneas de este escrito, las editoras hicieron el resumen y análisis de cada uno de los ensayos en la misma introducción de este libro, por lo tanto me propuse en esta presentación no reseñar ensayo por ensayo, sino dar un panorama de los temas abordados en los diversos trabajos que forman esta obra conjunta; quise también hacer una lectura en contraste-asociación-comparación de este libro, y encontré fuertes semejanzas; una de ellas fue, a diferencia de otras obras donde son evidentes las diferencias de profundidad y profesionalismo analítico, que cada una de las 12 autoras de estos ensayos hizo una clara delimitación de su tesis central y la abordó con rigor teórico y metodológico, ayudado por un vasto y adecuado manejo de fuentes que incluyeron no sólo buena parte de la producción del escritor(a) estudiado, además de la obra central de análisis, sino también otras que abordan el tema de cada ensayo.

Las 12 autoras muestran en esta obra que no son iniciadas en el campo de la literatura, en este caso de la crítica temática comparativa. Las diversas publicaciones anteriores de cada una de ellas muestran su experiencia y las aportaciones que han hecho en este campo de la ciencia.

Sobre la libertad que los autores estudiados y la temática seleccionada dio a cada ensayista, las 12 muestran de manera muy semejante el trabajo riguroso de análisis-síntesis que realizan, de tal manera que el resultado no fue un libro integrado por 12 partes, sino una unidad coherente que enriquece a quien lo lee, gracias a las diferencias, semejanzas y continuidades que lo cruzan, no sólo en los temas abordados por los escritores analizados y puestos en contraste, sino por las de las mismas autoras de este texto.

Elvia Montes de Oca Navas El Colegio Mexiquense, A.C. Correo-e: emontes@cmq.edu.mx

Elvia Montes de Oca Navas es doctora en estudios latinoamericanos por la Universidad Nacional Autónoma de México, miembro del Sistema Nacional de Investigadores. Actualmente trabaja como profesora-investigadora en El Colegio Mexiquense, A.C. Sus líneas de investigación son la historia bibliográfica del estado de México y la historia del estado de México durante el gobierno de Lázaro Cárdenas. Algunos de sus libros publicados son Protagonistas de las novelas de la Revolución Mexicana (1995), 
La educación socialista en el Estado de México. Una historia olvidada (1998), Imágenes del Estado de México 1824 (1999), Bibliografía especializada. Teotihuacan (1999), Politica laboral y corporativismo en el Estado de México 1934-1940 (2000), Sor Juana Inés de la Cruz. Bibliohemerografía (2002) y Los dueños y las tierras de la hacienda Santa Cruz de los Patos (2004). 